UMD-PP-98-112

SMU-98-03

\title{
Nucleosynthesis constraints on massive, stable, strongly interacting particles
}

\author{
Rabindra N. Mohapatra ${ }^{1}$ and Vigdor L. Teplitz ${ }^{2}$ \\ (1) Department of Physics, University of Maryland, College Park, Md-20742, USA. \\ (2) Department of Physics, Southern Methodist University, Dallas, Tx-75275, USA
}

\begin{abstract}
We find constraints on heavy, stable, strongly interacting massive particles (X) from searches for anomalous nuclei containing them, formed during primordial nucleosynthesis. Using existing data, we obtain a limit on the abundance ratio $C_{X} \equiv n_{X} / n_{B}$ in the range of $3 \times 10^{-8}$ to $3 \times 10^{-13}$ for masses up to $10 \mathrm{TeV}$ if the $X-N$ interaction is sufficiently strong to bind in low Z nuclei. We also find a rough lower limit on the $X-N$ interaction that implies binding in nuclei with $A \geq 200$ over much of the $M_{X}$ range of interest, and address the relative abundance of such anomalous nuclei on Earth.
\end{abstract}


Recently a number of authors have entertained the possibility that strongly interacting massive particles (SIMPS), with masses a few $\mathrm{GeV}$ and above, may play a role in particle physics and cosmology [1, 2, 3, 4, 5, 6, 7]. For example, these particles have been proposed as the source of ultra high energy cosmic rays (UHECR) by Chung et al. and others 2, 3]. There are interesting models of supersymmetry breaking where such particles appear as part of the messenger sector or as the gluino $\operatorname{LSP}[6]$.

Due to their strong interactions and high mass, these particles have distinct consequences in cosmology and astrophysics. In order to study their implications, it is essential to know their annihilation cross section, $\sigma_{X \bar{X}}$ which in turn determines their relic density. It has recently been noted [5] that, if we assume $\sigma_{X \bar{X}} \sim M_{X}^{-2}$, they can appear in sufficient abundance to saturate either the cosmic density or the galactic halo. Simple estimates of their relic density give

$$
\eta_{X} \equiv \frac{n_{X}}{n_{\gamma}} \simeq 5 \times 10^{3} \frac{M_{X}}{M_{P l}}
$$

For a $1 \mathrm{TeV} X$ particle, this gives the relative abundance $C_{X} \equiv \frac{n_{X}}{n_{B}} \simeq 5 \times 10^{-3}$ or $n_{X} \simeq 5 \times 10^{-10} / \mathrm{cm}^{3}$. For it to saturate the cosmic density, its mass must satisfy $M_{X} \geq 10^{3.5} \mathrm{GeV}$.

If in the process of cosmological evolution, the $X$ particles formed the gravitational potential well and thus became the halo dark matter, their halo number density would be considerably enhanced and become $n_{X} \simeq 3 \times 10^{-4} / \mathrm{cm}^{3}$ or so and known estimates of their density could be used to constrain their masses as functions of cross sections 1 , 河.

In [5], using the above form for the $X$ annihilation cross section as well as a simple QCD inspired ansatz for the $X-A$ cross it was shown that if the $X$ 's constituted the halo dark matter and their masses were above $100 \mathrm{GeV}$, they would be in conflict with the results from underground detectors looking for dark matter. 
The basic reason for this conclusion is that heavy nonrelativistic particles lose only a small fraction of their energy in elastic scattering and will therefore have to undergo a large number of collisions before they can slow down and get captured. Thus they should easily penetrate to reach the underground detectors but their signals have not been seen. There are also constraints[5] from the cosmological grand unified photon spectrum [8] arising from particle-anti-particle annihilation if one assumes either both appear in equal abundance or the $X$ particles are their own anti particles. Again this would rule out $m_{X} \geq 100 \mathrm{GeV}$ if one assumes the abundance to be halo density. Similar conclusions were also derived using the limits on anomalous heavy isotope abundances.

There have been several experimental searches [9, 10] to see whether these particles exist as dark matter and stringent limits have been placed on their masses and their interaction cross sections with matter. Other information on the possible existence of SIMPs used in both References [1] and [5] comes from terrestrial searches for anomalous heavy nuclei such as those in Ref. [11]. Specifically, the experiment of Hemmick et al [11] set stringent upper limits on the abundance of nuclei containing such heavy stable particles. These limits were used in Ref.'s[1, [] to exclude SIMPs above a mass of 1-10 TeV. In order to study this question, one needs to know the strength of $X-N$ binding potential $V_{X N}$, which is apriori unknown. One may either leave it as a free parameter as we do in the first part or use a "factorization" ansatz i.e. $\sigma_{X N}^{2}=\beta \sigma_{X \bar{X}} \sigma_{N N}$ which, using the fact that cross sections scale as the square of the potential can give a rough idea about the magnitude of $V_{X N}$ (as we do in the second part).

In this brief note, we present two new results: (A) the first uses an earlier investigation of primordial nucleosynthesis of (low Z) anomalous nuclei containing $X$ [12] for the case of sufficiently strong $X-N$ interaction to place limits on the 
$X$ abundance for the case when the annihilation cross section is assumed as in Ref.[5] and $V_{X N}$ is left arbitrary and (B) the second uses the above mentioned "factorization" ansatz for the annihilation cross section to determine the likelihood of relic $X \mathrm{~s}$ binding in high $\mathrm{Z}$ nuclei for weaker $X-N$ interaction (Binding is more likely for high $\mathrm{Z}$ nuclei since their larger radii imply lower kinetic energies.).

\section{A. Binding in low Z nuclei}

In Reference[12], Dicus and Teplitz calculated the abundance of anomalous $Z>1$ nuclei that would be produced by primordial nucleosynthesis if there existed a new neutral, stable, massive baryon $X^{0}$ ( the name used in 12 was $H$ ). This was motivated by earlier work of Dover, Gaisser and Steigman 13 on cosmological consequences of such particles. These papers assumed that the lightest color singlet bound state involving the massive colored particle and/or quarks and gluons interacts with nucleons in a similar way to that of the $\Lambda$ hyperon

The result of Reference [12] was an estimate of the abundances of anomalous nuclei relative to the total present abundance of all isotopes of $H e, L i, B e$, and $B$ for the case of low $\mathrm{Z}$ binding. It showed a significant enhancement in the abundance of ${ }^{9} B e^{*}$. Although ${ }^{8} B e$ is not stable, the $\Lambda$ hyperfragment ${ }^{9} B e_{\Lambda}^{*}\left({ }^{8} B e\right.$ to which a $\Lambda$ has been added) is stable. Reference [12] showed that replacing $\Lambda$ by another strongly

interacting particle, such as $X^{0}$, present with a cosmic abundance $C_{X} \equiv \frac{n_{X}}{n_{B}}=\frac{\eta_{X}}{\eta_{B}}$ at cosmic nucleosynthesis, would lead to a value for the ratio of the abundance ${ }^{9} \mathrm{Be}$ (anomalous ${ }^{9} \mathrm{Be}$ ) to the sum of all non-anomalous $\mathrm{Be}$ isotopes observed today greater than the ratio of the abundances of ${ }^{5} \mathrm{He}^{*}$ to ${ }^{4} \mathrm{He}$ by a factor of about $10^{4}$.

We make the natural assumption that any primordially synthesized anomalous heavy isotope should be present in random samples on earth in the same abundance as at the time of nucleosynthesis. However to be conservative, we will assume that $90 \%$ of any primordial ${ }^{9} B e^{*}$ will have been destroyed in stars. This is a conservative 
estimate because less than $90 \%$ of the Universe's Hydrogen has been cycled through stars; indeed interstellar deuterium (deuterium is also astrated) is of the order of one third the value of primordial calculations.

We consider (i) the question of what $X-$ Nucleus (also denoted by $X-A$ ) cross section we expect on the basis of our knowledge of the $\Lambda$ hyperfragment and (ii) whether for such cross sections, $X^{0}$ 's will be captured during cosmic nucleosynthesis. We then apply the constraint of Ref. [12] to limit either the $X-A$ cross section or the $X$ abundance in the light of the data of Ref.s [14, 15, 11].

Following the line of reasoning given for $\Lambda$ hyperfragments by Z. Povh [16], we use for the binding energy of $X$-particles in nuclei:

$$
B_{X}=V_{X}-\frac{\pi^{2}}{2 \mu R^{2}}
$$

where $V_{X}$ is the depth ( $A$-independent) of the potential well for the $X-A$ system, $R=R_{0}(A-1)^{1 / 3}$, and $\mu$ is the reduced mass for the $X-A$ system. We note here that $X-N$ binding has been studied in a very helpful paper by R. Plaga 17] for the case of $M_{X}<1 \mathrm{GeV}$. In the $\Lambda$ case, $R_{0}$ is about 1.5 Fermi and $V_{\Lambda} \simeq 27$ MeV. $B\left({ }^{5} \mathrm{He}_{\Lambda}\right)$ is about $3.1 \mathrm{MeV}$. Note that because of the absence of the one pion exchange force from the $\Lambda-N$ potential, there is no $\Lambda$ analog to the deuteron $\left({ }^{2} H_{\Lambda}\right)$ although ${ }^{3} H_{\Lambda}$ is bound. Based on Eq. (2), we would expect ${ }^{5} H e^{*}$ which is crucial to the formation of $B e^{*}$ and other light anomalous nuclei, to exist at the time of nucleosynthesis for some other strongly interacting particle $X$ provided the $X-N$ interaction is strong enough to produce an effective square well of depth of 10.5 $\mathrm{MeV}$. Here we have required that the binding energy $B_{X}$ be greater than $2.2 \mathrm{MeV}$ because primordial nucleosynthesis proceeds rapidly after the temperature becomes low enough that the high energy tail of the $\gamma$ distribution can no longer dissociate deuterium. The low energy (triplet) N-N cross section is about 4 barns whereas the low energy $\Lambda-N$ cross section is about 0.5 barns. Since the cross section goes as the 
square of the scattering amplitude which is proportional to the effective potential in some approximation, this implies if $\sigma(X N)$ is bigger than 0.1 barns, we expect to have a bound state. Although we cannot be certain about the modifications to Eq.(2) due to large $M_{X}$, it works well for hyperfragments and our intuition is that as the kinetic energy decreases relative to the potential due to larger $M_{X}$ the approximation should improve.

We turn next to the question of whether the $X$ particle will be captured by ${ }^{4} \mathrm{He}$ nuclei to form ${ }^{5} \mathrm{He} e^{*}$ nuclei. The probability of capture is roughly

$$
P=n_{\gamma 0} \eta_{B} 0.1\left(T / T_{0}\right)^{3} v t 10^{-24} \sigma_{b}
$$

where $n_{\gamma 0}$ is today's CBR density; $\eta_{B}$ is the baryon to photon ratio; the factor .1 takes into account account the Helium fraction relative to the nucleons; $\mathrm{T}$ and $\mathrm{t}$ are the temperature and time at the time of nucleosynthesis; $T_{0}$ is the CBR temperature today; and the relative velocity $v \simeq v_{H e} \simeq\left(6 T m_{H e}^{-1}\right)^{1 / 2} \simeq .1 c$ ( $c$ is the velocity of light). Finally $\sigma_{b}$ is the cross section in barns for $X$ to be captured by ${ }^{4} \mathrm{He}$. We compute $\sigma_{b}$ following Blatt and Weiskopf[18] on the electric dipole moment contribution to $n+p \rightarrow d+\gamma$ as discussed in Plaga[17]. For $X$-capture by a nucleus of atomic weight $\mathrm{A}$ and atomic number $\mathrm{Z}$. we have

$$
\sigma_{c a p}(A, Z, k) \simeq \frac{8 \pi \alpha Z^{2}}{3} \gamma^{-2}\left(\frac{k \gamma}{k^{2}+\gamma^{2}}\right)^{3}\left(\frac{\kappa}{k}\right)^{2}
$$

where $\gamma=\sqrt{(2 \mu B)}, k^{2} / 2 M_{X} \simeq .1 \mathrm{MeV}$ and $\kappa \simeq B$. Assuming $B \simeq 2.2 \mathrm{MeV}$, we estimate that $\sigma_{b} \simeq 3 \times 10^{-8}$ barns giving $P \sim 10^{-3}$ for large $M_{X}$ (even for $M_{X} \simeq M_{N}, P \simeq 0.1$. Note that this result corrects the assumption of Ref. 13. copied by Ref. [12] that $P \geq 1$. It shows that cosmic nucleosynthesis will not "hide" stable SIMPs in light nuclei so that signals from $X \bar{X}$ annihilation discussed in Ref. [5] and elswhere must be expected if there is no large $X-\bar{X}$ asymmetry. 
Let us now consider the limits on the abundance of the $X$ if the $X-N$ interaction is strong enough for formation of ${ }^{5} \mathrm{He}^{*}$ and ${ }^{9} \mathrm{Be} e^{*}$. To derive the abundance of ${ }^{9} \mathrm{Be} e^{*}$, we need to know the abundance of ${ }^{5} \mathrm{He}^{*}$ relative to normal ${ }^{4} \mathrm{He}$ at the time of nucleosynthesis since the reaction responsible for the synthesis of ${ }^{9} B e^{*}$ is ${ }^{4} \mathrm{He}\left({ }^{5} \mathrm{He} e^{*}, \gamma\right){ }^{9} \mathrm{Be} e^{*}$. One can then write

$$
\left.\frac{n_{9} B e^{*}}{n_{B e}}\right|_{\text {now }}=\left(\frac{n_{9} B e^{*}}{n_{5} H e^{*}}\right)\left(\frac{n_{5} H e^{*}}{n_{B}}\right)\left(\frac{n_{B}}{n_{B e}}\right)
$$

From Ref. [12], we get ${ }^{9} \mathrm{Be} e^{*}$ to ${ }^{5} \mathrm{He} e^{*}$ ratio to be $10^{-6}$; Eq. (3) tells us that the second factor in the above equation is $10^{-3} C_{X}$, and finally the present $B e$ abundance is taken fron Reeves 19] who gives $\frac{n_{B e}}{n_{B}} \simeq 2 \times 10^{-11}$. Combining gives the ratio ${ }^{9} B e^{*} / B e \simeq 50 C_{X}$. Other anomalous isotopes would have abundances below $C_{X}$ according to Ref. [12]. In order to reach these conclusions, Ref.[12] uses known rates for analog production and depletion reactions.

The data on $B e$ is summarized by Hemmick et al.[11] as follows: Klein et al [15] have ruled out isotopes of $B e$ up to $93 \mathrm{AMU}$ at concentrations above $10^{-12}$ per nucleon using accelerator mass spectrometry whereas Hemmick et al limit the abundance to $10^{-11}$ or less per nucleon for 100 to $1000 \mathrm{AMU}$ and to $10^{-9}$ or less up to 10,000 AMU ए. It should also be noted that Vanderschiff et al. 20 have placed limits on anomalous ${ }^{5} \mathrm{He}^{*}$ for $42 \leq M_{X} \leq 82$.

Taking into account the above result that $B e^{*} / B e \simeq 50 C_{X}$ and assuming $90 \%$ destruction of ${ }^{9} B e^{*}$ in stars means that the limits on $C_{X}$ are: $3 \times 10^{-13}$ to $M_{X} \leq 93$ $\mathrm{GeV}, 3 \times 10^{-12}$ for $100 \leq M_{X} / G e V \leq 1000$ and $3 \times 10^{-9}$ for $1000 \leq M_{X} / G e V \leq 5000$ and $3 \times 10^{-8}$ between $5 \leq M_{X} / T e V \leq 10$. These limits will apply to models in which a strongly interacting particle $X$ has an attractive interaction with nucleons with sufficient strength that the anomalous $B e^{*}$ forms with binding energy greater

\footnotetext{
1) Profs. Elmore and Hemmick have assured us that the results of Ref. 11] obtain down to 93 AMU
} 
than 2.2 MeV. Roughly such models should include cases in which $X-N$ scattering is attractive and $\sigma_{X N} \geq .1$ barns ${ }^{2}$.

\section{B. Binding in high Z nuclei}

We now consider the case in which $V_{X N}$ is too small for binding with $H e$ or $B e$. Even though we do not know the nature of the $X-N$ force from fundamental principles, we can put a lower bound on $V_{X N}$ using the afore mentioned "factorization" hypothesis i.e. $\sigma_{X N}^{2}=\beta \sigma_{X \bar{X}} \sigma_{N N}$ which gives

$$
\begin{array}{r}
V_{X N} \sim V_{N N}\left(\sigma_{X N} / \sigma_{N N}\right)^{1 / 2} \\
>V_{N N}\left[\left(\sigma_{X \bar{X}}^{m i n} \sigma_{N N}\right)^{1 / 2} / \sigma_{N N}\right]^{1 / 2} \beta^{1 / 4}
\end{array}
$$

For $\sigma_{X \bar{X}}^{\min }$, we use the fact that $X \bar{X}$ annihilation cross-section must be sufficiently large to ensure that today $M_{X} n_{X} \leq \rho_{c}$. Using standard methods $\mathbb{\|}$, we find $\sigma_{X-\bar{X}} \geq$ $3 \times 10^{-13}$ barns (independent of $M_{X}$ ) which implies $V_{X N} \simeq 20 \mathrm{KeV}$ if $V_{N N} \sim 50$ MeV. Eq. (2) then gives a condition for the existence of the bound state (in the approximation $\left.B_{X} \ll V_{X}\right)$ :

$$
0.425 A^{2 / 3} \beta^{-1 / 4} \frac{V_{X N}}{V_{N N}} \geq A^{-1}+M_{X}^{-1}
$$

where by $M_{X}$ we mean $M_{X}$ in GeV's. For $A \ll\left(M_{X} / \mathrm{GeV}\right)$, this yields $A^{5 / 3} \geq$ $2.3 \frac{V_{N N}}{V_{X N}} \beta^{1 / 4}$. This yields $A \simeq 183 \beta^{3 / 20}$ for $R_{0}=1.3$ Fermi and $\simeq 154 \beta^{3 / 20}$ for $R_{0}=1.5 \mathrm{Fermi}$. For the case where $A \gg\left(M_{X} / \mathrm{GeV}\right)$, we have instead the condition for bound state formation $A^{2 / 3} \geq \frac{\pi^{2}}{2 M_{X} R_{0}^{2}}$ which yields, $A \geq 427$ for $M_{X}=10 \mathrm{GeV}$. Bound states may exist for smaller $A$ and/or $M_{X}$ if $V_{X N}$ exceeds its minimum. It is important to note that the lower bound on $A$ is very insensitive to the value $\beta$ which characterizes any deviation from the formula $\sigma_{X N}^{2}=\sigma_{N N} \sigma_{X \bar{X}}$. If we set $A=238$ in

\footnotetext{
${ }^{2)}$ Note that if we used the "factorization" formula for cross sections and $V_{X N} \sim 10 \mathrm{MeV}$, we would obtain a much higher annihilation rate in the early universe and consequently a lower relic density $C_{X}$; nevertheless, it would still be inconflict with the bounds of Ref. [11], thus maintaining our conclusion.
} 
Eq. (8), we should be able to detect bound states of $X$ 's with minimal interaction down to $M_{X}=83 \mathrm{GeV}$.

We can again use Eq. (5) to compute the rate for $X$-capture. Assuming that $X$-particles have the galactic virial veloclty $\left(v \sim 10^{-3} c\right)$ and halo dark matter density

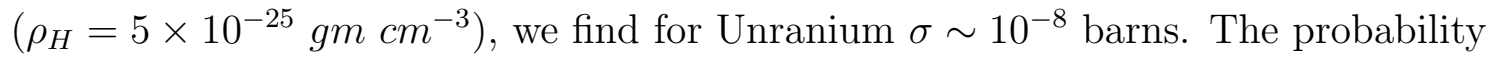

of $U$ capturing an $X$ in the lifetime of the solar system is then $P=\frac{\rho_{H}}{M_{X}} \sigma v_{v i r} \tau_{\text {solar }} \simeq$ $1.6 \times 10^{-11} /\left(M_{X}(T e V)\right)$.

One can extend the above line of reasoning to discuss what happens when the $X$ particles have nuclear cross sections necessary for them to play the role of UHECR's. Consider for example the case discussed in Ref. 3 where they need to have a $\sigma_{X N}$ of order of a milli-barn. Using our simple scaling laws, we conclude that this implies $V_{X N} \simeq 10^{-2} V_{N N}$. Inserting in Eq. (2) gives for large $M_{X}$ that bound states should exist for $A \geq 30$ (for $A \ll\left(M_{X} / G e V\right)$ ) assuming of course that the potential is attractive. Setting $A=238$ gives bound states for $M_{X}>6.3 \mathrm{GeV}$. In this case, the capture cross section ranges from $10^{-4} \mathrm{~b}$ for $M_{X}=6.3 \mathrm{GeV}$ to $10^{-7} \mathrm{~b}$ for large $M_{X}$ so that abundances are larger than minimal interaction case (assuming similar galactic halo dark matter).

To date searches for anomalous isotopes only seem to have reached $Z=9$ (Fluorine). Based on the above considerations, we urge experimentalists to search for anomalous isotopes with highest $\mathrm{Z}$ so that different interaction strengths for SIMPs may be explored. In particular, anomalous Uranium may provide a higher discovery potential if the halo dark matter is in fact dominated by "minimally" strongly interacting massive particles. However searches in much lighter elements $A \geq 30$ should stable SIMPs capable of explaining UHECRs.

\section{Acknowledgements}


The work of R. N. M. is supported by the National Science Foundation grant under no. PHY-9802551 and the work of V. L. T. is supported by the DOE under grant no. DE-FG03-95ER40908. We would like to thank R. Boyd, S. Brodsky, D. A. Dicus, D. Elmore, G. Farrar, E. Fishbach, T. Hemmick, Xiangdong Ji, E. W. Kolb, R. Middleton, G. Steigman and S. Wallace for discussions.

\section{References}

[1] G. Starkman, A. Gould, R. Esmilzadeh and S. Dimopoulos, Phys. Rev. D 41, 2388 (1990).

[2] D. Chung, G. Farrar and E. W. Kolb, FERMILAB-PUB-97/187; see also C. Albuquerque, G. Farrar and E. W, Kolb, astro-ph/9805288.

[3] V. Berezinsky and M. Kachelriess, hep-ph/9709485; V. Berezinsky and B. L. Ioffe, Sov. Phys. JETP 63, 920 (1986); for a review, see V. Berezinsky, hepph/9802351; M. Birkel and S. Sarkar, hep-ph/9804285.

[4] E. Nardi and E. Roulet, Phys. Lett. B245, 105 (1990); S. Chivukula et al. Phys. Rev. Lett. 65, 957 (1990).

[5] R. N. Mohapatra and S. Nussinov, Phys. Rev. D 57, 1940 (1997).

[6] Theoretical models that lead to stable massive strongly interacting particles are: R. N. Mohapatra and S. Nandi, Phys. Rev. Lett. 79, 181 (1997); Z. Chacko, B. Dutta, R. N. Mohapatra and S. Nandi, Phys. Rev. D 56, 5466 (1997); S. Raby, Phys. Rev. D 56, 2852 (1997); For tests of such models in colliders, see C. S. Li, P. Nadolsky, C. P. Yuan and H. Y. Zhou, hep-ph/9804258; H. Baer, K. Cheung and J. Gunion, hep-ph/9806361.

[7] D. Chung, E. W. Kolb and A. Riotto, hep-ph/9802238. 
[8] E. W. Kolb and M. Turner, Early Universe, Addison-Wesley, (1990).

[9] J. Rich et al. Phys. Lett. B 194, 173 (1987).

[10] P. C. McGuire et al., Arizona-IMAX collaboration preprint (1994); P. C. Mcguire, Ph. D. thesis, Univ. of Arizona (1994).

[11] T. K. Hemmick et al., Phys. Rev. D 41, 2074 (1990).

[12] D. A. Dicus and V. L. Teplitz, Phys. Rev. Lett. 44, 218 (1980).

[13] C. Dover, T. Gaisser and G. Steigman, Phys. Rev. Lett. 42, 1117 (1979); S. Wolfram, Phys. Lett. 82 B, 65 (1979).

[14] R. Middleton et al. Phys. Rev. Lett. 43, 429 (1979).

[15] R. Middleton et al. ANL-PHY-81-1 (1981).

[16] Z. Povh, Ann. Rev. Nucl. and Part. Sc. 28, 1 (1978).

[17] R. Plaga, Phys. Rev. D 51, 6504 (1995).

[18] J. A. Blatt and V. F. Weiskopf, Theoretical Nuclear Physics, Dover publications (1991).

[19] H. Reeves, Ann. Rev. Astron. and Astroph., 12, 437 (1974).

[20] J. Vandegriff, G. Raimann, R. N. Boyd and P. Ruiz, Phys. Lett. B 365, 418 (1996). 